\title{
OPEN Afidopyropen, a novel insecticide originating from microbial secondary extracts
}

\author{
Ryo Horikoshi ${ }^{1 凶}$, Kimihiko Goto ${ }^{1}$, Masaaki Mitomi ${ }^{1}$, Kazuhiko Oyama ${ }^{1}$, Tomoyasu Hirose ${ }^{2}$, \\ Toshiaki Sunazuka² \& Satoshi Ōmura² \\ Afidopyropen, a novel insecticide, is a derivative of pyripyropene $A$, which is produced by the \\ filamentous fungus Penicillium coprobium. Afidopyropen has strong insecticidal activity against aphids \\ and is currently used as a control agent of sucking pests worldwide. In this study, we summarized the \\ biological properties and field efficacies of its derivatives against agricultural pests using official field \\ trials conducted in Japan. Afidopyropen showed good residual efficacies against a variety of aphids, \\ whiteflies and other sucking pests under field conditions. Furthermore, toxicological studies revealed \\ its safety profiles against nontarget organisms, such as the honeybee, natural enemies and other \\ beneficial insects, as well as mammals. Thus, afidopyropen is a next-generation agrochemical for crop \\ protection that has a low environmental impact.
}

Many pharmaceuticals and agrochemicals have been discovered from natural sources, such as extracts of plants and microbes, and they play important practical roles under field conditions. In agriculture, more than $20 \%$ of the agrochemical market consists of natural products, semi-synthetic products derived from natural compounds and biomimetic compounds ${ }^{1,2}$, and new agrochemical discoveries related to crop protection are still being reported ${ }^{3}$. Our group has focused on screening microbial extracts to discover new drugs, including pesticidal compounds ${ }^{4-7}$. Through the active screening of natural sources, including purified chemicals, we identified pyripyropene A (PP-A) in the Meiji natural compounds library. It was isolated as a compound that inhibited the activity of acylCoA:cholesterol acyltransferase ${ }^{8-12}$ and possessed high insecticidal activity against aphids. Aphids cause damage to crops, resulting in production losses worldwide, by feeding on plant phloem sap and vectoring a variety of viruses that cause destructive plant diseases ${ }^{13}$. As an insecticide, PP-A has a unique chemical structure with 3 -pyridyl, $\alpha$-pyrone and sesquiterpene moieties, and in aphids, exposure results in strong disorientation that ultimately leads to death ${ }^{14}$. Although PP-A showed good activity in laboratory assays, the residual activity in field trials was unexpectedly shorter than that of commercial standards ${ }^{15}$. To improve the residual efficacy in the field, we elucidated the SAR using the Kitasato's pyripyropene derivatives library and structural optimization. After the synthesis of various derivatives, an early SAR study that focused on aphids dropping off treated leaves in laboratory assays revealed that the symptom occurred after exposure to derivatives having highly lipophilic substituents, including second lead compound 1 (Fig. 1). This compound produces a better dermal activity in foliar laboratory assays than PP-A; however, a consistent field efficacy was not achieved ${ }^{16}$. Therefore, as a next step, we focused on improving the oral activities of the PP-A derivatives that were ingested by aphids through the sucking of plant phloem sap, and afidopyropen emerged as a candidate insecticide. It had a log P value of 3.45 and water solubility of $25.1 \mathrm{mg} / \mathrm{L}$. It is more hydrophilic than compound 1, which had a $\log \mathrm{P}$ value of 4.8 and water solubility of $0.4 \mathrm{mg} / \mathrm{L}^{17}$. A key substituent, hydroxyl, at the C7 position markedly increased not only the insecticidal activity against aphids but also the systemic activity. Afidopyropen had more than 60 times lower $\mathrm{LC}_{90}$ than PP-A in Myzus persicae (Table 1), shifting $\mathrm{LC}_{90}$ values from 0.45 to $0.0068 \mathrm{ppm}$, and it also exhibited excellent residual efficacy in field trials. Currently, many insecticides, such as organophosphates (OPs), carbamates, pyrethroids, neonicotinoids, ketoenols and pyrazoles, are available ${ }^{18}$ and are used as tools to control sucking pests. However, resistance problems to some of these insecticides have emerged in target insect pests. In addition, some insecticides are being banned or their use has been strictly limited owing to their undesirable impacts on honeybees, beneficial insects or other nontarget organisms. Therefore, new eco-friendly insecticides that aid in achieving sustainable agriculture are strongly required. Afidopyropen has been launched globally in countries such as the USA, India, China and Australia under the brand name Inscalis insecticide by BASF

\footnotetext{
${ }^{1}$ Agricultural and Veterinary Research Labs., Agricultural and Veterinary Division, Meiji Seika Pharma Co., Ltd., 760 Morooka-cho, Kohoku-ku, Yokohama 222-8567, Japan. ${ }^{2}$ Graduate School of Infection Control Sciences, Ōmura Satoshi Memorial Institute, Kitasato University, Tokyo, Japan. ${ }^{\circledR}$ email: Ryou.Horikoshi@mitsuichemicals.com
} 


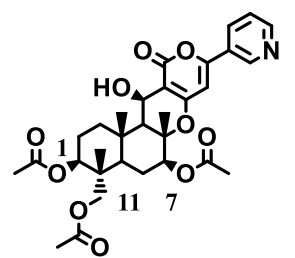

Pyripyropene A

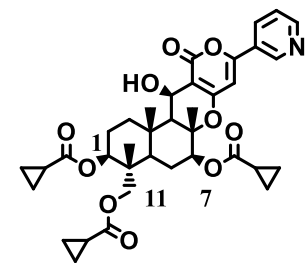

1

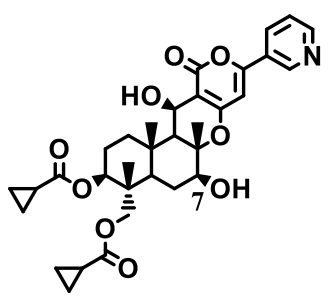

Afidopyropen

Figure 1. Discovery of afidopyropen.

\begin{tabular}{|c|c|c|c|c|c|}
\hline \multirow[b]{2}{*}{ Order } & \multirow[b]{2}{*}{ Scientific name } & \multirow[b]{2}{*}{ Growth stage } & \multicolumn{3}{|l|}{$\mathrm{LC}_{90}(\mathrm{mg} / \mathrm{L})$} \\
\hline & & & Afidopyropen & PP-A & Cpd. 1 \\
\hline \multirow{19}{*}{ Hemiptera } & \multirow{2}{*}{ Myzus persicae } & \multirow{2}{*}{ 1st instar larva } & 0.0068 & 0.45 & 0.022 \\
\hline & & & $\left(0.001768<{ }^{*}<0.02611\right)$ & (N.D.) & $\left(0.01443<^{*}<0.03401\right)$ \\
\hline & \multirow{2}{*}{ Aphis gossypii } & \multirow{2}{*}{ 1st instar larva } & 0.012 & 0.242 & 0.090 \\
\hline & & & $\left(0.009029<<^{*}<0.01666\right)$ & $(188.5<*<309.9)$ & $\left(0.02759<^{*}<0.2906\right)$ \\
\hline & \multirow{2}{*}{ Aphis craccivora } & \multirow{2}{*}{ Mix of all stages } & 2.9 & \multirow{2}{*}{-} & \multirow{2}{*}{-} \\
\hline & & & (N.D.) & & \\
\hline & \multirow{4}{*}{ Trialeurodes vaporariorum } & \multirow{2}{*}{ Adult } & 2.6 & 7.8 & - \\
\hline & & & $\left(1.447<^{*}<4.839\right)$ & $(3.782<*<15.99)$ & \\
\hline & & \multirow{2}{*}{ Egg } & 0.13 & \multirow{2}{*}{-} & \multirow{2}{*}{-} \\
\hline & & & $\left(0.0565<<^{\star}<0.307\right)$ & & \\
\hline & \multirow{4}{*}{ Bemisia tabaci } & \multirow{2}{*}{ Biotype B, Adult } & 1.5 & \multirow{2}{*}{-} & \multirow{2}{*}{-} \\
\hline & & & $(1.064<*<2.050)$ & & \\
\hline & & \multirow{2}{*}{ Biotype Q, Adult } & 2.1 & 2.6 & 5.8 \\
\hline & & & (N.D.) & (N.D.) & (N.D.) \\
\hline & \multirow{2}{*}{ Pseudococcus comstocki } & \multirow{2}{*}{ 1st instar larva } & 0.3 & 12.1 & 1.3 \\
\hline & & & $\left(0.126<^{*}<0.650\right)$ & (N.D.) & $\left(1.145<^{*}<1.451\right)$ \\
\hline & \multirow{2}{*}{ Empoasca onukii } & \multirow{2}{*}{ Adult } & 17 & \multirow{2}{*}{-} & \multirow{2}{*}{-} \\
\hline & & & $\left(7.365<^{*}<38.76\right)$ & & \\
\hline & Nilaparvata lugens & 2nd instar larva & $>100$ & $>100$ & $>100$ \\
\hline \multirow{2}{*}{ Lepidoptera } & \multirow{2}{*}{ Plutella xylostella } & \multirow{2}{*}{ 2nd instar larva } & \multirow{2}{*}{$>100$} & & 10 \\
\hline & & & & $>100$ & $\left(6.058<^{*}<16.3\right)$ \\
\hline Thysanoptera & Frankliniella occidentalis & 1st instar larva & $>100$ & $>100$ & $>100$ \\
\hline Diptera & Liriomyza trifolii & Adult & $>100$ & - & - \\
\hline Coleoptera & Oulema oryzae & Adult & $>1000$ & - & - \\
\hline Acari & Tetranychus urticae & Egg & $>100$ & $>500$ & $>100$ \\
\hline
\end{tabular}

Table 1. Insecticidal spectrum of afidopyropen against agricultural pests. -, not tested; (), 95\% confidence interval; N.D. no data.

(Ludwigshafen, Germany), and it is expected to address the above problems while relieving negative impacts on agricultural ecosystems. Here, we summarize its insecticidal properties and effects on nontarget organisms.

\section{Results}

Insecticidal spectra. In our study, afidopyropen showed excellent insecticidal activities against common aphid species, such as green peach (Myzus persicae), cotton (Aphis gossypii) and bean (Aphis craccivora), that damage a variety of vegetables, fruit trees, tea trees and ornamentals by sucking sap from sprouts and leaves. Furthermore, afidopyropen showed good activities against whiteflies (Trialeurodes vaporariorum and Bemisia tabaci Biotype Q), mealybugs (Pseudococcus comstocki), leafhoppers (Empoasca onukii) and psyllids, a hemipteran insect, and it exhibited good efficacies against these insect pests in field trials, while decreasing crop damage. These pests are common on many crops, such as cotton, beans and vegetables, and some pests have developed resistance to existing insecticides. Afidopyropen demonstrated good to excellent efficacy against multiple life stage of T. vaporariorum, B. tabaci Biotype Q and E. onukii. An ovicidal efficacy was not observed, but afidopyropen showed good activities against T. vaporariorum and B. tabaci Biotype $\mathrm{Q}$ after they hatched. It did not show insecticidal activities against Lepidoptera (Plutella xylostella), Thysanoptera (Frankliniella occidentalis), Diptera (Liriomyza trifolii), Coleoptera (Oulema oryzae) and Acari (Tetranychus urticae), indicating selectivity against 


\begin{tabular}{|c|c|c|c|}
\hline & \multicolumn{3}{|l|}{$\mathrm{LC}_{90}$} \\
\hline & Susceptible & OP resistant ${ }^{*}$ & Neonicotinoids resistant ${ }^{\star}$ \\
\hline \multirow{2}{*}{ Afidopyropen } & 0.012 & 0.008 & 0.002 \\
\hline & $\left(0.001768<<^{*}<0.02611\right)$ & (N.D.) & (N.D.) \\
\hline \multirow{2}{*}{ Imidacloprid } & 0.1638 & 0.2014 & 10.33 \\
\hline & $\left(141.3<^{*}<189.9\right)$ & $\left(0.06553<{ }^{*}<0.619\right)$ & $\left(6.705<^{*}<15.92\right)$ \\
\hline \multirow{2}{*}{ Acephate } & 3.9 & \multirow{2}{*}{$>100$} & 87 \\
\hline & $\left(0.987<^{*}<15.07\right)$ & & (N.D.) \\
\hline
\end{tabular}

Table 2. Efficacy of a foliar afidopyropen application against cotton aphid field populations. ${ }^{*}$ The populations collected in Japan fields. N.D. no data.

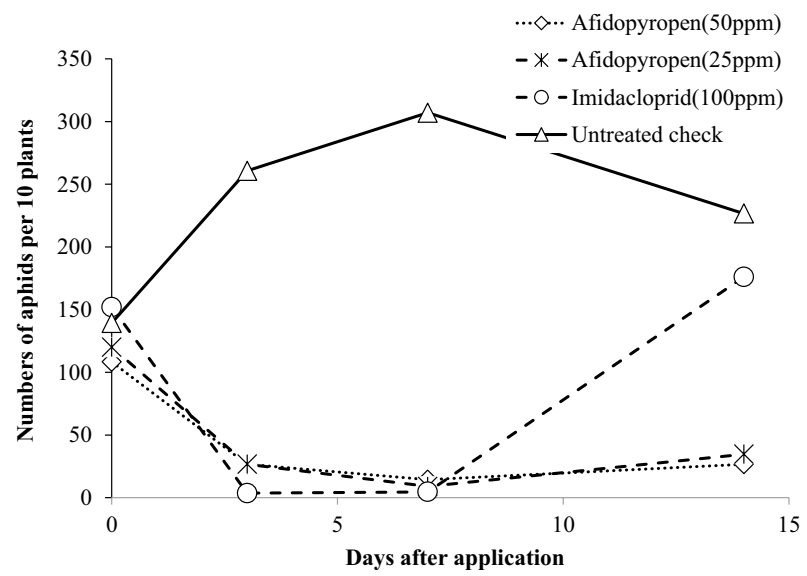

Figure 2. Field efficacy of foliar afidopyropen applications against cotton aphids on potato.

\begin{tabular}{|l|l|l|l|l|}
\hline Generation & $\mathbf{L C}_{\mathbf{5 0}}(\mathbf{m g} / \mathbf{L})$ & $\mathbf{9 5} \%$ confidence interval & $\mathbf{L C}_{\mathbf{9 0}}(\mathbf{m g} / \mathbf{L})$ & 95\% Confidence interval \\
\hline F0 & 0.00380 & $0.002752<^{*}<0.005234$ & 0.0184 & $0.009112<^{*}<0.03706$ \\
\hline F10 & 0.00726 & $0.005835<^{*}<0.009035$ & 0.0196 & $0.01411<^{*}<0.02719$ \\
\hline
\end{tabular}

Table 3. Efficacy of afidopyropen against the F0 (unselected) and F10 population selected by a $0.002 \mathrm{ppm}$ afidopyropen spray.

Hemipteran pests (Table 1). Similarly, PP-A and compound 1 exhibited excellent efficacies against M. persicae and $A$. gossypii and moderate efficacies against $P$. comstock $i$ and adult whiteflies. Their insecticidal spectra were the same as that of afidopyropen, except some activity observed for compound $\mathbf{1}$ against $P$. xylostella.

Insecticidal activity against resistant insect pests. Aphids populations resistant to commercial insecticides are appearing worldwide. Some aphids are developing resistance to OP or neonicotinoids, which are widely used to control sucking pests. Therefore, we tested the efficacy of afidopyropen against resistant populations of cotton aphids collected in Japan. Afidopyropen showed excellent efficacy against both OP and neonicotinoid resistant cotton aphids collected in fields that were equivalent or superior to its efficacy against a susceptible population (Table 2). These finding demonstrate a lack of cross resistance to OP and neonicotinoids.

In addition to its excellent insecticidal efficacy against resistant populations as determined by laboratory assays, afidopyropen showed good efficacy towards populations with reduced susceptibility to commercial standards in official field trials conducted by the Japan Plant Protection Association (JPPA). In the trials, afidopyropen was used as a foliar spray at lower concentrations than imidacloprid (Fig. 2). Moreover, cotton aphids were subjected to continued selection using a foliar afidopyropen spray to assess the risk of developing afidopyropen resistance (Table 3). After breeding for 10 generations, the differences between both the $\mathrm{LC}_{50}$ and $\mathrm{LC}_{90}$ for afidopyropen were less than twofold between the F0 and F10.

Speed of control. Aphids and whiteflies damage many crops by vectoring viruses that cause diseases. Therefore, it is essential for insecticides to quickly prevent sucking pests from transmitting viral diseases. Consequently, we evaluated the effects of a temporary exposure to a $10 \mathrm{ppm}$ foliar afidopyropen spray of cucumber 


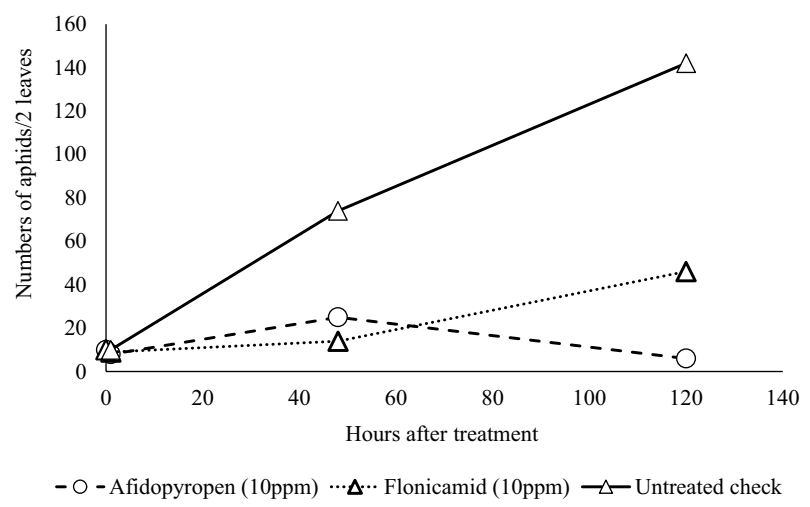

Figure 3. Efficacy of an afidopyropen application against adult cotton aphids after a 1-h exposure.

\begin{tabular}{|l|l|l|l|}
\hline \multirow{4}{*}{ Treatment } & & Numbers of aphids & \\
\cline { 2 - 4 } Afidopyropen & Concentration $(\mathbf{p p m})$ & Treated pot & \% Control \\
\hline & 25 & 0 & 100 \\
\cline { 2 - 4 } & 12.5 & 32 & 76 \\
\cline { 2 - 4 } & 6.25 & 24 & 82 \\
\hline Untreated check & & 135 & - \\
\hline
\end{tabular}

Table 4. Translaminar efficacy of afidopyropen against green peach aphid on eggplant. At 7 days after treatment of only the upside of a true leaf with each afidopyropen concentration, the number of aphids on the opposite untreated side was counted.

\begin{tabular}{|c|c|c|c|c|}
\hline \multirow[b]{2}{*}{ Systemicity } & \multirow[b]{2}{*}{ Leaf observed } & \multicolumn{2}{|c|}{ Numbers of aphids } & \multirow[b]{2}{*}{$\%$ Control } \\
\hline & & Treated pot & Untreated pot & \\
\hline \multirow{2}{*}{ Upward } & 2nd true leaf & 1 & 162 & 99.4 \\
\hline & 1st true leaf* & 7 & 225 & 96.9 \\
\hline \multirow{2}{*}{ Downward } & 2nd true leaf* & 20 & 369 & 94.6 \\
\hline & 1st true leaf & 136 & 343 & 60.3 \\
\hline
\end{tabular}

Table 5. Systemicity of topical afidopyropen application against cotton aphids on cucumber. ${ }^{\star}$ Treated leaves. At 4 days after treatment of only the 1 st or 2 nd true leaf with 1,000 ppm afidopyropen, adult cotton aphids were placed on cucumber plants. After $8 \mathrm{~d}$, the numbers of aphids were counted.

leaf disks on adult aphids. After a 1-h exposure, the increase in the number of aphids discontinued, and afidopyropen's efficacy was equivalent to that of the common commercial standard flonicamid (Fig. 3).

Systemicity of afidopyropen on crops. Given that aphids prefer to infest new sprouts and young undeveloped leaves, it is a desired attribute for aphid-controlling agrochemicals to penetrate from treated leaves to untreated young leaves or to untreated parts of leaves systemically within the plants. In tests to assess its systemicity from treated to untreated leaf surfaces, afidopyropen showed a good translaminar efficacy against the green peach aphid providing moderate to excellent control (Table 4). Furthermore, the upward systemicity from a treated to an untreated cucumber leaf was demonstrated with afidopyropen exhibiting over $95 \%$ aphid control on both leaves. However, the downward systemicity from a treated to an untreated downward leaf was lower with afidopyropen exhibiting $60.3 \%$ aphid control (Table 5). Because commercial standards for aphid control are frequently used in systemic applications, such as soil drenching or nursery box application in young seedlings, the root systemicity was also evaluated in a field trial. As a soil drenching agent, $20 \mathrm{mg}$ afidopyropen per seedling showed a good systemicity against cotton aphids on cucumber, but the efficacy was inferior to imidacloprid (Fig. 4). Furthermore, the seed-treatment efficacy was investigated by dipping wheat seeds into the insecticide solution. Similar to soil drenching tests, afidopyropen exhibited good efficacy to wheat aphid when seeds were dipped into $500 \mathrm{ppm}$ of afidopyropen solution for $6 \mathrm{~h}$ (Fig. 5). Although the systemicity from roots and seeds were moderate in field trials (data not shown), afidopyropen possessed good systemicity in crops and controlled aphids through some exposure routes.

Globally, low water volumes are commonly used when spraying pesticides. To confirm its efficacy under such conditions, we evaluated the effects of spraying at a low volume (400 L/ha) of $320 \mathrm{mg} / \mathrm{L}$ afidopyropen and at a 


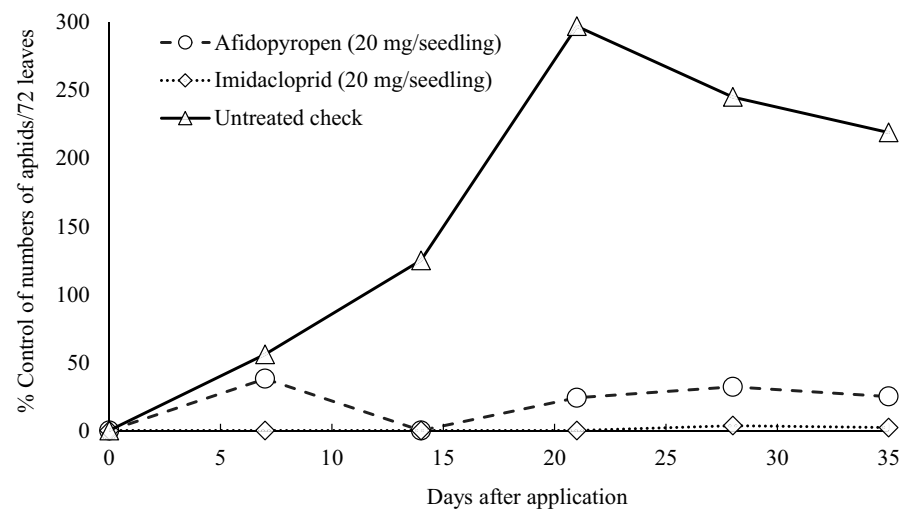

Figure 4. Field efficacy of afidopyropen against cotton aphids on cucumber at 7, 14,21, 28 and 35 days after soil drenching with afidopyropen or imidacloprid. Afidopyropen or imidacloprid was placed in each transplantation hole, and the numbers of aphids naturally infesting the plants were counted at each time point.

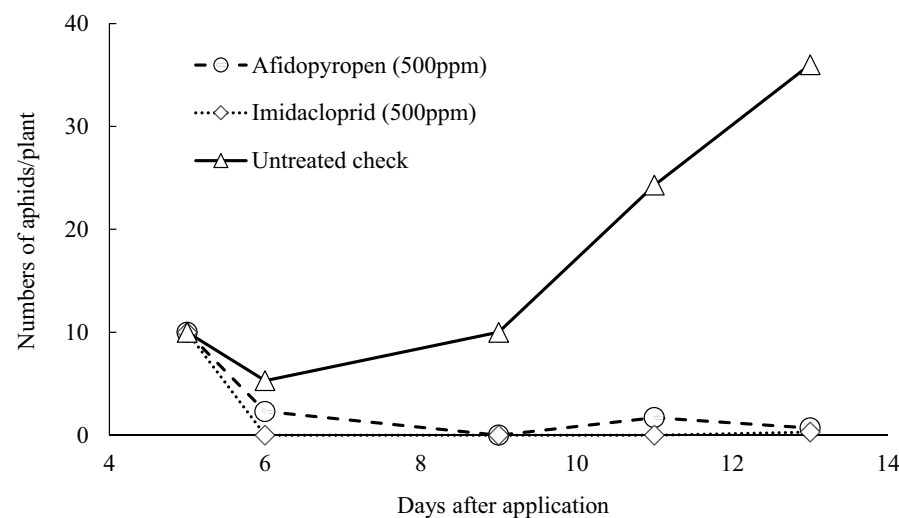

Figure 5. Efficacy of afidopyropen against wheat aphid by seed dipping. At 5 days after seed dipping, 10 young wheat aphid larvae were placed on each plant. At 1, 4, 6 and 8 days after infestation, the numbers of aphids were counted.

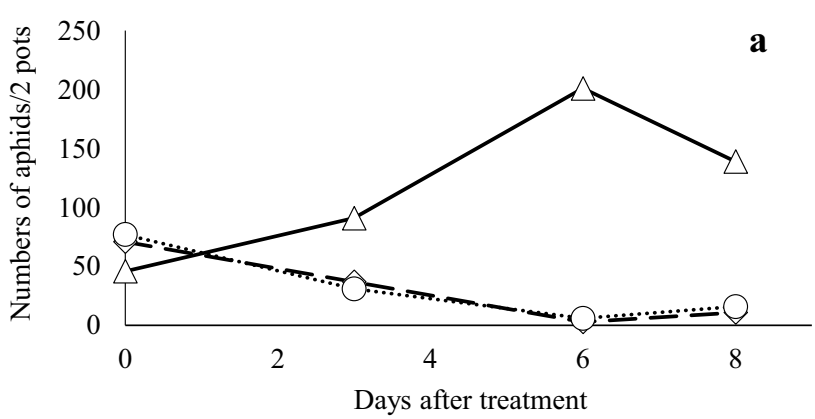

$\longrightarrow$ - Afidopyropen (low volume) $\cdots \circ \cdots$ Afidopyropen (high volume) $\rightarrow$ Untreated check

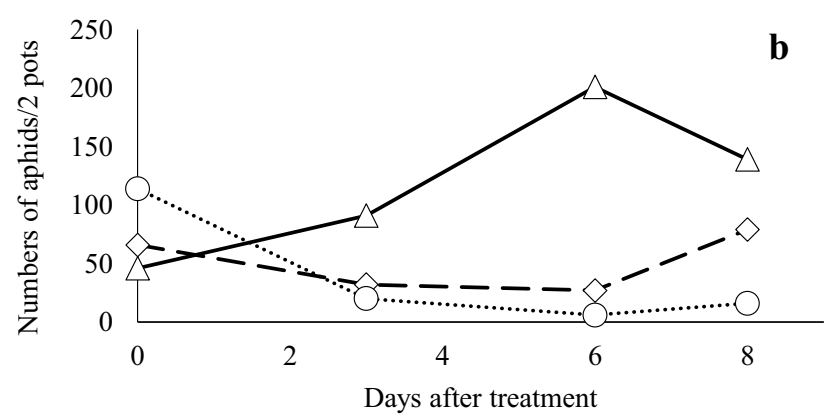

$\longrightarrow$ - Flonicamid (low volume) $\cdots \circ \cdots$ Flonicamid (high volume) $\rightarrow$ Untreated check

Figure 6. Efficacy of insecticides against wheat aphid at low (400 L/ha of $320 \mathrm{mg} / \mathrm{L}$ pesticide) and high (1280 L/ ha of $100 \mathrm{mg} / \mathrm{L}$ pesticide) water volumes. (a) Afidopyropen; (b) flonicamid.

normal volume (1280 L/ha) of $100 \mathrm{mg} / \mathrm{L}$ afidopyropen. The efficacy was the same under both conditions, and the spray volume did not affect the efficacy (Fig. 6a), which was slightly better than that of flonicamid (Fig. 6b).

Management of viral spread. Through the JPPA, we conducted field trials of an afidopyropen formulation containing 10\% technical grade of active ingredient against sucking pests, and it showed good residual efficacies against aphids on vegetables and fruit trees for more than 2 weeks after a foliar application. In soy- 


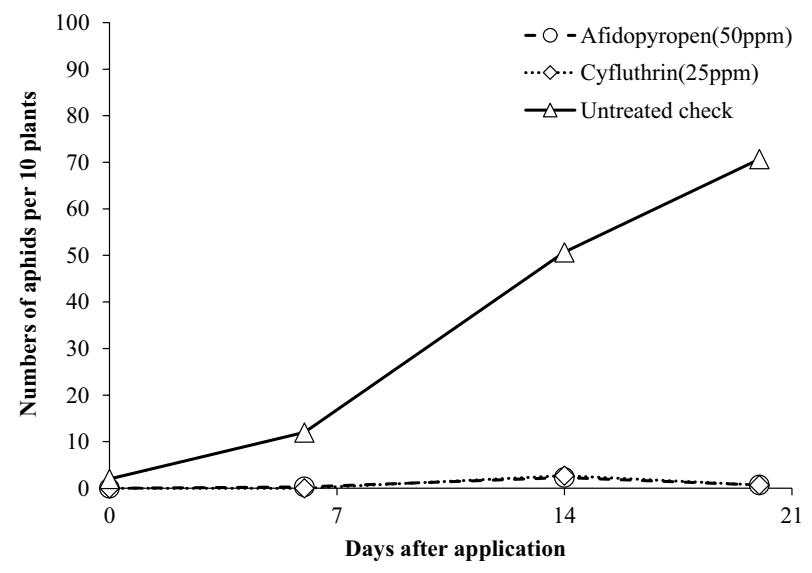

Figure 7. Control efficacies of weekly 4 times foliar afidopyropen applications on soybeans against greenhousepotato aphids in a 2010 field test conducted by the Hokkaido Plant Protection Association.

\begin{tabular}{|l|l|l|}
\hline Species & $\mathbf{L C}_{\mathbf{5 0}}$ or $\mathbf{E C}_{\mathbf{5 0}}$ or $\mathbf{E r C}_{\mathbf{5 0}}(\mathbf{m g} / \mathbf{L})$ & $\mathbf{9 5} \%$ Confidence interval \\
\hline Cyprinus carpio & 18 & $15<^{*}<23$ \\
\hline Daphnia magna & 8.0 & $5.0<^{*}<15$ \\
\hline Pseudokirchneriella subcapitata & $>25$ & N.D. \\
\hline
\end{tabular}

Table 6. Effects of afidopyropen on aquatic organisms. N.D. no data.

\begin{tabular}{|l|l|l|l|}
\hline Species & Application & \multicolumn{2}{l|}{ Mortality } \\
\hline Apis mellifera in labs & Oral & $\mathrm{LD}_{50}$ & $>100 \mu \mathrm{g} / \mathrm{bee}$ \\
\hline Apis mellifera in labs & Contact & $\mathrm{LD}_{50}$ & $>100 \mu \mathrm{g} / \mathrm{bee}$ \\
\hline Apis mellifera in semi field & Foliar application & $\mathrm{LD}_{50}$ & $>116.7 \mathrm{gai} / \mathrm{ha}$ \\
\hline
\end{tabular}

Table 7. Effects of afidopyropen on the honeybee. ${ }^{\star}$ At $3,8,24$ and $48 \mathrm{~h}$ after alfalfa was treated with afidopyropen, 25 worker honeybees were released into a chamber containing collected alfalfa foliage. The deaths in each chamber were observed at 3,24 and $48 \mathrm{~h}$ after release. Each test had six replicates.

bean field trials, we confirmed that 4 times 50 ppm afidopyropen foliar spray not only decreased the numbers of greenhouse potato aphids on the soybeans 6,14 and 20 days after 1st application (Fig. 7), it also inhibited the incidence of the dwarfing disease caused by the aphid vector $62 \mathrm{~d}$ after 1 st application. Thus, afidopyropen $(50 \mathrm{ppm})$ significantly controlled soybean dwarf virus (SbDV) transmission by $27 \%$ in infested plants compared with untreated plants, as well as decreasing the numbers of aphids.

Mode of action. Afidopyropen does not act on the nicotinic acetylcholine, GABA, glutamate, octopamine and serotonin receptors, nor on sodium channels. Furthermore, the inhibition of acetylcholine esterase, the mitochondrial electron transfer system and insect growth regulator is also not observed ${ }^{19}$. A recent study revealed that afidopyropen modulates the transient receptor potential vanilloid (TRPV) channels in insect chordotonal organs $\mathrm{s}^{20}$. Chordotonal organs, which are unique to insects and crustaceans, are mechano-sensors, which are located in the joints of body segments and provide information about relative rotation of body parts. Hyperactivation and eventual silencing of TRPV channels by afidopyropen perturbs function of chordotonal organs and leads to loss of coordination, which, in turn results in inability to feed, desiccation and eventual death. Afidopyropen is classified into the 9D group of pyropene chemistry on insecticide classification by the Insecticide Resistance Action Committee ${ }^{21}$.

Regarding crop safety, $10 \%$ afidopyropen in water-dispersible granules has been used in a variety of Japanese field trials to confirm the insecticidal efficacy of this formulation. At an effective dose rate of $50 \mathrm{ppm}$, the foliar spray did not show any toxic effects against cereals, vegetables, tea trees, fruit trees or ornamentals.

The toxicity levels of afidopyropen against aquatic invertebrates (Table 6), honeybees (Table 7) and a variety of beneficial insects (Table 8) were low ${ }^{22}$. In the USA and EU, issues relating to the bee toxicity levels of existing agrochemicals are important, but a bee study using laboratory assays and semi-field trials revealed that 


\begin{tabular}{|l|l|l|l|}
\hline Species & Application & Mortality \\
\hline Harmonia axyridis & Contact & $\mathrm{LD}_{50}$ & $>100 \mathrm{mg} / \mathrm{L}$ \\
\hline Chrysoperla carnea & Contact & $\mathrm{LD}_{50}$ & $>100 \mathrm{mg} / \mathrm{L}$ \\
\hline Aphidius colemani & Contact & $\mathrm{LD}_{50}$ & $>100 \mu \mathrm{g} / \mathrm{cm}^{2}$ \\
\hline Encarsia formosa & Contact & $\mathrm{LD}_{50}$ & $>100 \mu \mathrm{g} / \mathrm{cm}^{2}$ \\
\hline Orius strigicollis & Contact & $\mathrm{LD}_{50}$ & $>100 \mathrm{mg} / \mathrm{L}$ \\
\hline Pardosa astrigera & Contact & $\mathrm{LD}_{50}$ & $>100 \mathrm{mg} / \mathrm{L}$ \\
\hline Episyrphus balteatus & Contact & $\mathrm{LD}_{50}$ & $>100 \mathrm{mg} / \mathrm{L}$ \\
\hline Aphidoletes aphidimyza & Contact & $\mathrm{LD}_{50}$ & $>100 \mu \mathrm{g} / \mathrm{cm}$ \\
\hline Anisodactylus signatus & Contact & $\mathrm{LD}_{50}$ & $>100 \mathrm{mg} / \mathrm{L}$ \\
\hline Eisenia foetida & Contact & $\mathrm{LD}_{50}$ & $>1000 \mathrm{mg} / \mathrm{kg} \mathrm{soil}$ \\
\hline
\end{tabular}

Table 8. Effects of afidopyropen on beneficial organisms. The acute toxicity to all beneficial organisms was evaluated in accordance with the guidelines in the Appendix to Director General Notification, No. 12-Nousan-8147, 24 November, 2000, Agricultural Production Bureau, Ministry of Agriculture, Forestry and Fisheries of Japan or OECD No.207 guideline.

\begin{tabular}{|l|l|}
\hline Study & Effect \\
\hline Acute oral toxicity to rat & $\mathrm{LD}_{50}>2000 \mathrm{mg} / \mathrm{kg}$ \\
\hline Acute dermal toxicity to rat & $\mathrm{LD}_{50}>2000 \mathrm{mg} / \mathrm{kg}$ \\
\hline Acute inhalation toxicity to rat & $\mathrm{LD}_{50}>5.48 \mathrm{mg} / \mathrm{L}$ \\
\hline Acute neurotoxicity to rat & NOEL $200 \mathrm{mg} / \mathrm{kg}$ \\
\hline Sub-acute toxicity to rat in 90 days & NOEC $300 \mathrm{ppm}$ \\
\hline Chronic toxicity to rat in 1 year & NOEC $300 \mathrm{ppm}$ \\
\hline Carcinogenic toxicity to rat in 2 years & NOEC $300 \mathrm{ppm}$ \\
\hline 2 generations reproductive toxicity to rat & NOEC $300 \mathrm{ppm}$ \\
\hline Developmental toxicity to rat & NOEL $30 \mathrm{mg} / \mathrm{kg} /$ day \\
\hline Ames test & Negative \\
\hline Skin irritation to rabbit & No irritation \\
\hline Eye irritation to rabbit & Minimally irritating \\
\hline
\end{tabular}

Table 9. Mammalian toxicity studies using afidopyropen.

afidopyropen has limited toxic effects. The mammalian toxicity studies revealed no serious acute, sub-acute or other toxicity issues (Table 9).

\section{Discussion}

In this study, we revealed that afidopyropen had excellent insecticidal activity against devastating piercing and sucking agricultural pests, although its insecticidal spectrum was narrow. The narrow spectrum might result from the modes of action of the TRPV modulators that help insects recognize outside mechanical stimuli and maintain proper posture and behavior in response to the stimuli, and we observed only weak activity with Cpd. 1 on Plutella xylostella lepidopteran larvae. The efficacy differs among the insect stages. Since hemipteran larvae are aggressively seeking foliage for feeding, the efficacy is especially high. A behavioral abnormality has also been observed in some adult hemipteran insect species, and it halts the population's growth. Hemipteran pests damage almost all crops, genetically modified and non-genetically modified, worldwide, and even with existing insecticides, they are still not sufficiently controlled. Drug resistance and/or registration issues owing to toxicological and eco-toxicological properties of existing insecticides have negatively impacted control strategies. In particular, those of the main target pest, the aphid, with its short lifecycle and ability to quickly develop insecticide resistance. In fact, the development of resistance against major insecticides, like neonicotinoids, is remarkable ${ }^{23,24}$. Afidopyropen has a novel chemical scaffold and does not show cross resistance with major insecticides such as OPs, synthetic pyrethroids and neonicotinoids. In some target insects, like whitefly, even pymetrozine, which acts on TRPV, the same target protein as afidopyropen, have resistance issues develop owing to metabolic factors, but afidopyropen shows a good efficacy against pymetrozine-resistant whiteflies ${ }^{25}$. Moreover, our selection study using a foliar afidopyropen spray showed a low risk of resistance development. Afidopyropen is an insecticide effective by foliar spray, not only in high volume, but also in low volume applications which are globally used practices. Afidopyropen has good translaminar efficacy and systemic activity and has suitable systemic properties to control sucking pests that prefer new shoots and seedlings. In addition, afidopyropen has good control efficacies against aphids when used in soil drench and seed dip applications, as well as a good control efficacy against whitefly when used in soil drench (data not shown). However, the dose rate is higher than those of standards. Further investigations would be needed to find suitable useful scenarios in systemic uses. Although the speed of 
kill was slow, insects treated with afidopyropen showed abnormal behaviors in a few hours and became unable to damage plants in a short time. In fact, in some field trials, treatments stopped the development of diseases resulting from aphid-vectored viruses and very few remaining dead insects were observed because they easily fell off the treated leaves. As documented in Japanese official field trials on a variety of crops, afidopyropen has shown excellent efficacy for the control of aphids, whiteflies, leafhoppers and mealybugs.

As well, regarding consistency of efficacy across the pest spectrum overseas, afidopyropen exhibits excellent field efficacy against aphid, whitefly and the Asian citrus psyllid at low doses, 10-50 gai/ha, by BASF ${ }^{26-30}$, and the Food Safety Commission of Japan information indicates that it is not persistent in the environment ${ }^{22}$. For instance, the $\mathrm{DT}_{50}$ for afidopyropen is 2.7-18.6 days in soils under aerobic conditions, and 1-2 months under simulated sunlight. After application to crops, it is relatively labile with low persistence. The main metabolite, its dimer, has low acute and sub-acute toxicity levels as well.

In addition, afidopyropen shows low toxicity levels against honeybees and natural enemies, as well as against mammals. Because of environmental dynamics, afidopyropen is expected to be an eco-friendly tool of sustainable agriculture. Now that afidopyropen has been launched by BASF SE and entered into the crop protection market worldwide, it will aid in achieving sustainable agriculture. Moreover, we seek opportunities to extend this technology into unexplored crop production segments and application scenarios to improve current practices and contribute to enhanced crop productivity, including systemic uses and the treatment to control other pests and synergistic combinations.

\section{Methods}

General. Afidopyropen was produced and purified in accordance with our established methods ${ }^{31}$. The commercial insecticides imidacloprid and flonicamid were purchased from FUJIFILM Wako Pure Chemical Corporation (Tokyo, Japan). Organic solvents, chemical reagents and all the consumables were purchased from FUJIFILM Wako Pure Chemical Corporation (Osaka, Japan).

The authors confirm that we used plants commercially available in Japan in this research and all studies involving plants and plant materials were done in compliance with local and national regulations/guidelines.

Insecticidal assays. Laboratory insecticidal tests against agricultural pests. Afidopyropen was evaluated using each target insect pest on leaf disks or potted plants in accordance with our previous report ${ }^{32}$. The test conditions are summarized in the Supplementary Information section. Resistant populations and populations present on $0.002 \mathrm{ppm}$ afidopyropen (50\% acetone/distilled water containing $0.05 \%$ Tween 20 ) solution-treated cucumber leaves were also tested with cotton aphids using the same leaf disk assays. Cabbage (Kinkei 201, purchased from Sakata), cucumber (Suyo, purchased from Sakata), fava bean (Funaokaissun, purchased from Takayama seed), kidney bean (Celina, purchased from Takii), wheat (Nourin 61, seeds collected inhouse), rice (Jikkoku, seeds collected inhouse) or tea (Yabukita planted inhouse) plants were used for each test, which are available in Japan. $\mathrm{LC}_{90}$ was calculated in a probit method with ECOTOX v3 software.

Speed of control assay. Adult cotton aphids were exposed to glass dishes treated with each insecticide after drying for $1 \mathrm{~h}$. Then, five treated adults were placed on a cucumber (Suyo) leaf disks. At 1, 48, 120 and $168 \mathrm{~h}$ after infestation, the numbers of live adults and larvae were counted. The test was conducted with two replicates.

Translaminar assay. At 7 days after treating only the upside of an eggplant (Senryou 2, purchased from Takii) true leaf with each afidopyropen solution concentration, four adults of green peach aphids were placed on the opposite untreated leaves of the $2.8 \mathrm{~cm}$ diameter of leaves cut from the treated eggplants. The number of aphids on the opposite untreated sides was counted. The test was conducted with three replicates.

Systemic activity from treated leaves. At 4 days after treating only the 1 st or 2 nd cucumber (Suyo) true leaf with 1000-ppm afidopyropen, three adult cotton aphids were placed on the leaves. The numbers of aphids on both the treated and untreated upward/downward leaves were counted after 8 days. The test was conducted with two replicates.

Soil drenching efficacy. When transplanting 3-week-old cucumber (Suyo) seedlings, afidopyropen formulation or a commercial insecticide imidacloprid was applied into each transplantation hole. At 7, 14, 21, 28 and 35 days after transplantation, the numbers of aphids on 72 leaves from 12 plants were counted. The test was conducted in triplicate, with 12 plants per replicate.

Seed dipping efficacy. The wheat (Nourin 61) seeds were dipped in a water-based dilution of 5\% wettable afidopyropen powder for $6 \mathrm{~h}$. After the treatment, seeds were allowed to put up shoots for $72 \mathrm{~h}$. Then, the seeds were transplanted. At 2 days after transplantation, 10 adult wheat aphids were placed onto the seedlings. At 1, 4, 6 and 8 days after infestation, the number of aphids was counted in each plot. This test was conducted in triplicate.

Efficacy of foliar applications against cotton aphid (A. gossypii) on potato. A JPPA field trial was conducted in 2011 using 5-week-old potato (Nishiyutaka, available in Japan) plants. A solution of afidopyropen dissolved in water was applied at $2000 \mathrm{~L} /$ ha to potatoes naturally infested with the cotton aphid. At 3, 7 and 14 days after application, the number of aphids was counted on 10 plants in each plot. Each plot was tested in triplicate. 
Corrected density index $=[$ (number of aphids on treated plant at X days after application $) /$ (number of aphids on treated plant before application)] $\times[$ (number of aphids on untreated plant before application $) /$ (number of aphids on untreated plant at X days after application)] $\times 100$.

Then, compared with the untreated plants, the control rate was calculated as follows:

$$
\text { control rate }(\%)=100-(\text { corrected density index }) \text {. }
$$

Management of viral spread. A field trial was conducted in 2010 by the Hokkaido Plant Protection Association using approximately 2-month-old soybean (Toyomusume, available in Japan) plants. A solution of afidopyropen dissolved in water was applied at $150 \mathrm{~L} /$ ha to soybeans naturally infested with the glasshouse potato aphid Aulacorthum solani. At 3, 7 and 13 days after application, the number of aphids was counted on 10 plants in each plot. Furthermore, the number of plants infested by SbDV was counted 13 days after application. Each test was conducted in triplicate. The control rate (\%) for infested aphids was calculated using Eq. (2), and SbDV infection (\%) and control rate (\%), compared with untreated plants, was calculated using the following formulae:

$$
\begin{aligned}
\operatorname{SbDV} \text { rate }(\%)= & (\text { number of infected plants on treated plant at } 13 \text { days after application }) \\
& /(\text { number of infected untreated plants atX days after application }) \times 100 .
\end{aligned}
$$

and

$$
\text { control rate }(\%)=100-(\text { infected rate of SbDV). }
$$

Received: 1 July 2021; Accepted: 2 February 2022

Published online: 18 February 2022

\section{References}

1. Charles, L. C., Franck, E. D. \& Stephen, O. D. Natural products as sources for new pesticides. J. Nat. Prod. 75, 1231-1242 (2012).

2. Sparks, T. C., Wessels, F. J., Lorsbach, B. A., Nugent, B. M. \& Watson, G. B. The new age of insecticide discovery-the crop protection industry and the impact of natural products. Pestic. Biochem. Phys. 161, 12-22 (2019).

3. Lorsbach, B. A. et al. Natural products: A strategic lead generation approach in crop protection discovery. Pest Manag. Sci. 75, 2301-2309 (2019).

4. Sasaki, T. et al. A new anthelmintic cyclodepsipeptide, PF1022A. J. Antibiot. 45, 692-697 (1992).

5. Gomi, S. et al. PF1018, a novel insecticidal compound produced by Humicola sp. J. Antibiot. 47, 571-580 (1994).

6. Goto, K. et al. Novel insecticidal/miticidal agent. WO 2006/137389 A1 (Meiji Seika Kaisha Ltd., 2006).

7. Takeuchi, H. et al. Plant parasitic nematode controlling agent and parasitic nematode controlling method. WO 2020/241702 A1 (Meiji Seika Pharma Co., Ltd., 2020).

8. Ōmura, S., Tomoda, H., Kim, Y. K. \& Nishida, H. Pyripyropenes, highly potent inhibitors of acyl-CoA:cholesterol acyltransferase produced by Aspergillus fumigatus. J. Antibiot. 46, 1168-1169 (1993).

9. Tomoda, H., Kim, Y. K., Nishida, H., Masuma, R. \& Ōmura, S. Pyripyropenes, novel inhibitors of acyl-CoA:cholesterol acyltransferase produced by Aspergillus fumigatus. I. Production, isolation, and biological properties. J. Antibiot. 47, 148-153 (1994).

10. Kim, Y. K. et al. Pyripyropenes, novel inhibitors of acyl-CoA:cholesterol acyltransferase produced by Aspergillus fumigatus. II. Structure elucidation of pyripyropenes A, B, C and D. J. Antibiot. 47, 154-162 (1994).

11. Tomoda, H. et al. Pyripyropenes, novel inhibitors of acyl-CoA:cholesterol acyltransferase produced by Aspergillus fumigatus. III. Structure elucidation of pyripyropene E to L. J. Antibiot. 48, 495-503 (1995).

12. Tomoda, H. et al. Pyripyropenes, novel ACAT inhibitors produced by Aspergillus fumigatus. IV. Structure elucidation of pyripyropene M to R. J. Antibiot. 49, 292-298 (1996).

13. Sorensen, J. T. Aphids, Chapter 8. In Encyclopedia of Insects 2nd edn (eds Resh, V. H. \& Carde, R. T.) 27-31 (Elsevier Inc., 2009).

14. Horikoshi, R. et al. Identification of pyripyropene A as a promising insecticidal compound in a microbial metabolite screening. J. Antibiot. 70, 272-276 (2017).

15. Horikoshi, R. et al. Insecticidal properties of pyripyropene A, microbial secondary metabolite, against agricultural pests. J. Pestic. Sci. 43, 266-271 (2018).

16. Goto, K. et al. Synthesis and insecticidal efficacy of pyripyropene derivatives focusing on the C-1, C-7, and C-11 positions' substituent groups. J. Antibiot. 71, 785-797 (2018).

17. Goto, K. et al. Synthesis and insecticidal efficacy of pyripyropene derivatives Part II-Invention of afidopyropen. J. Antibiot. 72, 661-681 (2019).

18. Phillips, M. W. A. Agrochemical industry development, trends in R\&D and the impact of regulation. Pest Manag. Sci. 76, 3348-3356 (2020).

19. Leichter, C. A., Thompson, N., Johnson, B. R. \& Scott, J. G. The high potency of ME-5343 to aphids is due to a unique mechanism of action. Pestic. Biochem. Physiol. 107, 169-176 (2013).

20. Kandasamy, R. et al. Afidopyropen: New and potent modulator of insect transient receptor potential channels. Insect Biochem. Mol. Biol. 84, 32-39 (2017).

21. Insecticide Resistance Action Committee (IRAC): IRAC Mode of Action Classification Scheme Version 9.1 (2018).

22. https://www.fsc.go.jp/iken-bosyu/pc1_no_afidopyropen_310206.data/pc1_no_afidopyropen_310206.pdf (2019).

23. Bass, C. et al. The evolution of insecticide resistance in the peach potato aphid, Myzus persicae. Insect Biochem. Mol. Biol. 51, 41-51 (2014).

24. Fernández, E. et al. Insecticide resistance status of Bemisia tabaci Q-biotype in south-eastern Spain. Pest Manag. Sci. 65, 885-891 (2009).

25. Nesterov, A. et al. Afidopyropen: New and potent modulator of insect TRP channels. In American Chemical Society Annual Meeting, Washington, DC, (2017).

26. Stout, J., Nyoike T., \& Salgado, V. Inscalis ${ }^{\text {Tw }}$ Insecticide: A novel insecticide for management of piercing-sucking pests. In Entomological Society of America Annual Meeting, Denver (2017). 
27. Nyoike T. et al. Inscalis ${ }^{\mathrm{TM}}$ Insecticide: A management tool for asian citrus psyllid (Diaphorina citri) in citrus. In Entomological Society of America Annual Meeting, Denver (2017).

28. Nyoike T. et al. Inscalis ${ }^{\text {Tm }}$ Insecticide for control of piercing-sucking pests in vegetable crops. In Entomological Society of America Annual Meeting, Denver (2017).

29. Keese R. et al. Ventigra ${ }^{\text {Tx }}$ Insecticide for control of piercing and sucking insects in ornamentals and vegetable transplants. In Entomological Society of America Annual Meeting, Denver (2017).

30. Nyoike T., Tiwari, S., \& Wofford, J. Inscalis ${ }^{\mathrm{Th}}$ Insecticide: A new insecticide for management of sap feeding insects in vegetables and tree crops. In Entomological Society of America Annual Meeting, St. Louis (2019).

31. Goto, K. et al. Pest control agent WO 2008/066153 A1 (Meiji Seika Kaisha Co., Ltd. \& the Kitasato Institute, 2008).

32. Kagabu, S., Mitomi, M., Kitsuda, S., Horikoshi, R., Nomura, M., \& Onozaki, Y. Pest Control Agent US Patent Appl. US9073866 (Meiji Seika Pharma Co., Ltd., 2013).

\section{Acknowledgements}

We appreciate BASF members for kind comments and revisions to this manuscript and Edanz (https://jp.edanz. com/ac) for editing a draft of this manuscript. Also, we thank Meiji members involved in the studies shown in this manuscript.

\section{Author contributions}

R.H. and K.O. had a responsibility for biological evaluation of afidopyropen. K.G. supported the preparation of this manuscript from the viewpoint of chemistry. And, M.M., T.H., T.S. and S.Ō. reviewed this manuscript and greatly supported to compose this manuscript.

\section{Competing interests}

The authors declare no competing interests.

\section{Additional information}

Supplementary Information The online version contains supplementary material available at https://doi.org/ 10.1038/s41598-022-06729-z.

Correspondence and requests for materials should be addressed to R.H.

Reprints and permissions information is available at www.nature.com/reprints.

Publisher's note Springer Nature remains neutral with regard to jurisdictional claims in published maps and institutional affiliations.

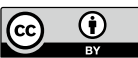

Open Access This article is licensed under a Creative Commons Attribution 4.0 International License, which permits use, sharing, adaptation, distribution and reproduction in any medium or format, as long as you give appropriate credit to the original author(s) and the source, provide a link to the Creative Commons licence, and indicate if changes were made. The images or other third party material in this article are included in the article's Creative Commons licence, unless indicated otherwise in a credit line to the material. If material is not included in the article's Creative Commons licence and your intended use is not permitted by statutory regulation or exceeds the permitted use, you will need to obtain permission directly from the copyright holder. To view a copy of this licence, visit http://creativecommons.org/licenses/by/4.0/.

(C) The Author(s) 2022 